\title{
A THEOREM FOR A FLUID SPHERE IN STOKES FLOW
}

\author{
D. PALANIAPPAN ${ }^{1}$, S. D. NIGAM ${ }^{1}$ \\ and T. AMARANATH ${ }^{1}$
}

(Received 7 February 1992; revised 1 October 1992)

\begin{abstract}
A sphere theorem for non-axisymmetric Stokes flow of a viscous fluid of viscosity $\mu^{e}$ past a fluid sphere of viscosity $\mu^{i}$ is stated and proved. The existing sphere theorems in Stokes flow follow as special cases from the present theorem. It is observed that the expression for drag on the fluid sphere is a linear combination of rigid and shear-free drags.
\end{abstract}

\section{Introduction}

The study of the hydrodynamic forces on viscous drops moving in an ambient fluid has various applications to problems such as dispersion of one fluid in another.

The Stokes flow due to uniform translation of a spherical fluid particle in an unbounded fluid medium was treated by both Rybczynski [15] and Hadamard [6]. Using Lamb's [10] general solution, Hetsroni and Haber [8] have found the solution for the flow fields, interior and exterior to a single spherical droplet submerged in an unbounded arbitrary Stokes flow. They have also obtained the expression for the drag on the droplet, which is a generalisation of Faxen's law for a rigid spherical particle. This result for drag was also derived by Rallison [14] using the reciprocal theorem. Making use of Lamb's [10] solution Fuentes et al. [3, 4] have studied the images of singularities such as Stokeslet and the higher order Stokes singularities near a viscous drop. As Lamb's [10] solution is in the form of an infinite series, the algebra in $[3,4]$ is highly involved and

\footnotetext{
${ }^{1}$ School of Math. and Comp./Inf. Sc., University of Hyderabad, India.

(C) Australian Mathematical Society, 1994, Serial-fee code 0334-2700/94
} 
the solution obtained is not in a closed form.

Shail [16] and Shail and Onslow [17] have proved sphere theorems for a class of non-axisymmetric flows within and past a rigid sphere respectively. They have employed a representation for velocity and pressure used in a paper by Hackborn et al. [5]. However, this analysis is restricted to the cases where the velocity dependence on the azimuthal angle, $\phi$, is linear in $\cos \phi$ and $\sin \phi$. Essentially, in this case, the problem reduces to an equivalent axisymmetric problem and the solution is readily obtained using Collin's [2] theorem and Butler's [1] theorem.

Recently, Palaniappan et al. [13] have given a general representation of the solution of Stokes equations in a closed form in terms of two scalar functions $\mathrm{A}$ and $\mathrm{B}$ which are biharmonic and harmonic respectively. It is also shown that this solution is equivalent to Lamb's [10] general solution and a theorem for non-axisymmetric Stokes flow past a rigid sphere is proved. In [12] a sphere theorem for non-axisymmetric Stokes flow about a shear-free sphere is proved.

In this note, we prove a sphere theorem for a general non-axisymmetric Stokes flow in and around a fluid sphere, by using the velocity representation given in [13]. The flow fields interior and exterior to a fluid sphere are given in a closed form in terms of the two scalar functions A and B. From this theorem the results of $[13,12]$ are deduced as special cases. The corresponding results for the axisymmetric case are presented as a corollary to the theorem. The expression for the drag on the fluid sphere is given and it is found to be a linear combination of rigid and shear-free drags. Several illustrative examples are presented to demonstrate the usefulness of our method and in each case the force acting on the fluid spherical particle is calculated.

\section{The problem}

We consider the steady flow of a viscous, incompressible fluid (viscosity $\mu^{e}$ ) past a viscous fluid sphere (viscosity $\mu^{i}$ ). The Stokes equations are as follows: Exterior to the fluid sphere

$$
\begin{aligned}
\mu^{e} \nabla^{2} \mathbf{q}^{e} & =\operatorname{grad} p^{e}, \\
\nabla \cdot \mathbf{q}^{e} & =0
\end{aligned}
$$

In the fluid sphere

$$
\begin{aligned}
\mu^{i} \nabla^{2} \mathbf{q}^{i} & =\operatorname{grad} p^{i}, \\
\nabla \cdot \mathbf{q}^{i} & =0,
\end{aligned}
$$


where $\mathbf{q}^{e}$ and $\mathbf{q}^{i}$ are the velocities outside and in the fluid sphere respectively, and $p^{e}$ and $p^{i}$ are the pressures. From [13], the velocity and pressure fields are as follows: Outside the fluid sphere

$$
\begin{aligned}
\mathbf{q}^{e} & =\operatorname{curl} \operatorname{curl}\left(\mathbf{r} A^{e}\right)+\operatorname{curl}\left(\mathbf{r} B^{e}\right), \\
p^{e} & =p_{0}+\mu^{e} \frac{\partial}{\partial r}\left(r \nabla^{2} A^{e}\right),
\end{aligned}
$$

where

$$
\begin{aligned}
& \nabla^{4} A^{e}=0 \\
& \nabla^{2} B^{e}=0 .
\end{aligned}
$$

In the fluid sphere

$$
\begin{aligned}
\mathbf{q}^{i} & =\operatorname{curl} \operatorname{curl}\left(\mathbf{r} A^{i}\right)+\operatorname{curl}\left(\mathbf{r} B^{i}\right), \\
p^{i} & =p_{0}+\mu^{i} \frac{\partial}{\partial r}\left(r \nabla^{2} A^{i}\right)
\end{aligned}
$$

where

$$
\begin{aligned}
\nabla^{4} A^{i} & =0, \\
\nabla^{2} B^{i} & =0 .
\end{aligned}
$$

From (5) the components of $\mathbf{q}^{e}$ in the directions of $r, \theta, \phi$ are respectively

$$
\begin{aligned}
q_{r}^{e} & =-\frac{1}{r}\left(\cot \theta A_{\theta}^{e}+A_{\theta \theta}^{e}+\operatorname{cosec}^{2} \theta A_{\phi \phi}^{e}\right), \\
q_{\theta}^{e} & =\frac{1}{r} \frac{\partial}{\partial \theta}\left(A^{e}+r A_{r}^{e}\right)+\operatorname{cosec} \theta B_{\phi}^{e} \\
q_{\phi}^{e} & =\frac{1}{r \sin \theta} \frac{\partial}{\partial \phi}\left(A^{e}+r A_{r}^{e}\right)-B_{\theta}^{e} .
\end{aligned}
$$

The components of $\mathbf{q}^{i}$ can be written down by replacing the superscript $e$ by $i$ in $(11 \mathrm{a}, \mathrm{b}, \mathrm{c})$. The boundary conditions are

(I): the normal velocity vanishes at the surface of the sphere, i.e.,

$$
q_{r}^{e}=q_{r}^{i}=0 \text { on } \quad r=a .
$$

(II): the tangential velocity components are continuous at the interface i.e.,

$$
q_{\theta}^{e}=q_{\theta}^{i}, \quad q_{\phi}^{e}=q_{\phi}^{i} \quad \text { on } \quad r=a .
$$


(III): the tangential stress components are continuous across the boundary i.e.,

$$
T_{r \theta}^{e}=T_{r \theta}^{i}, \quad T_{r \phi}^{e}=T_{r \phi}^{i} \quad \text { on } \quad r=a,
$$

where

$$
\begin{aligned}
& T_{r \theta}^{e}=\frac{\mu^{e}}{r^{2}} \frac{\partial}{\partial \theta}\left[r q_{r}^{e}+r^{2} \frac{\partial^{2} A^{e}}{\partial r^{2}}-2 A^{e}\right]+\mu^{e} r \operatorname{cosec} \theta \frac{\partial}{\partial \phi}\left[\frac{\partial}{\partial r}\left(\frac{B^{e}}{r}\right)\right], \\
& T_{r \phi}^{e}=\frac{\mu^{e}}{r^{2} \sin \theta} \frac{\partial}{\partial \phi}\left[r q_{r}^{e}+r^{2} \frac{\partial^{2} A^{e}}{\partial r^{2}}-2 A^{e}\right]-\mu^{e} r \frac{\partial}{\partial \theta}\left[\frac{\partial}{\partial r}\left(\frac{B^{e}}{r}\right)\right] .
\end{aligned}
$$

$T_{r \theta}^{i}$ and $T_{r \phi}^{i}$ can be obtained similarly be replacing ' $e$ ' by ' $i$ '. The above conditions are satisfied if, on $r=a$, we have

$$
\begin{aligned}
& A^{e}=A^{i}=0, \\
& A_{r}^{e}=A_{r}^{i} ; \quad B^{e}=B^{i}, \\
& \mu^{e} A_{r r}^{e}=\mu^{i} A_{r r}^{i} ; \quad \mu^{e} \frac{\partial}{\partial r}\left(\frac{B^{e}}{r}\right)=\mu^{i} \frac{\partial}{\partial r}\left(\frac{B^{i}}{r}\right) .
\end{aligned}
$$

\section{The theorem}

THEOREM. Let the flow of a viscous, incompressible fluid (with viscosity $\mu^{e}$ ) in the absence of any boundary be characterized by a biharmonic $A_{0}(r, \theta, \phi)$ and a harmonic $B_{0}(r, \theta, \phi)$. Let the singularities (We extend the term 'singularity' to include flows non-vanishing at infinity and regular everywhere else, such as uniform flow, quadratic flow etc.) of these functions be at a distance greater than ' $a$ ' from the origin. If $A_{0} \sim \mathrm{o}(r)$ and $B_{0} \sim \mathrm{o}(1)$ at the origin and if now a fluid (with viscosity $\mu^{i}$ ) sphere $r=a$ be introduced in the flow field with $q_{r}^{e}=q_{r}^{i}=0, q_{\theta}^{e}=q_{\theta}^{i}, q_{\phi}^{e}=q_{\phi}^{i}, T_{r \theta}^{e}=T_{r \theta}^{i}$ and $T_{r \phi}^{e}=T_{r \phi}^{i}$ on the boundary $r=a$, then the solution for $r>a$ is

$$
\begin{aligned}
A^{e}(r, \theta, \phi)= & A_{0}(r, \theta, \phi)+(1-\lambda)\left[-\frac{r}{a} A_{0}\left(\frac{a^{2}}{r}, \theta, \phi\right)\right] \\
& +\lambda\left[-\frac{\left(r^{2}+a^{2}\right)}{2 a r} A_{0}\left(\frac{a^{2}}{r}, \theta, \phi\right)+\frac{\left(r^{2}-a^{2}\right)}{a} \frac{\partial}{\partial r} A_{0}\left(\frac{a^{2}}{r}, \theta, \phi\right)\right. \\
& \left.-\frac{r^{2}\left(r^{2}-a^{2}\right)^{2}}{4 a^{3}} \nabla^{2}\left(r^{-1} A_{0}\left(\frac{a^{2}}{r}, \theta, \phi\right)\right)\right]
\end{aligned}
$$




$$
\begin{aligned}
& B^{e}(r, \theta, \phi)= B_{0}(r, \theta, \phi)+\lambda\left[-\frac{a}{r} B_{0}\left(\frac{a^{2}}{r}, \theta, \phi\right)\right] \\
&+(1-\lambda)\left[\frac{a}{r} B_{0}\left(\frac{a^{2}}{r}, \theta, \phi\right)-\frac{3 r}{a^{3}} \int_{0}^{a^{2} / r} R B_{0}(R, \theta, \phi) d R\right] \\
&+3(1-\lambda) \frac{r}{a^{3}}\left[\int_{0}^{a^{2} / r} R B_{0}(R, \theta, \phi) d R\right. \\
&\left.-(1-2 \lambda) \frac{a^{6 \lambda}}{r^{3 \lambda}} \int_{0}^{a^{2} / r} R^{1-3 \lambda} B_{0}(R, \theta, \phi) d R\right]
\end{aligned}
$$

and the solution for $r<a$ is

$$
\begin{aligned}
A^{i}(r, \theta, \phi)= & (1-\lambda)\left[\frac{\left(a^{2}-r^{2}\right)}{2 a^{2}} A_{0}(r, \theta, \phi)+\frac{r\left(r^{2}-a^{2}\right)}{a^{2}} \frac{\partial}{\partial r} A_{0}(r, \theta, \phi)\right. \\
\left.\quad-\frac{\left(r^{2}-a^{2}\right)^{2}}{4 a^{2}} \nabla^{2} A_{0}(r, \theta, \phi)\right] & \\
B^{i}(r, \theta, \phi)= & 2(1-\lambda) B_{0}(r, \theta, \phi) \\
& -3(1-\lambda)(1-2 \lambda) r^{3 \lambda-2} \int_{0}^{r} R^{1-3 \lambda} B_{0}(R, \theta, \phi) d R
\end{aligned}
$$

where $\lambda=\mu^{i} /\left(\mu^{e}+\mu^{i}\right)$.

PROOF. By a direct verification, it can be shown that the expressions given in (15)-(18) satisfy $(7 \mathrm{a}, \mathrm{b})$ and $(10 \mathrm{a}, \mathrm{b})$ together with the boundary conditions $(12 \mathrm{a}, \mathrm{b})-(14 \mathrm{a}, \mathrm{b})$. It is noted that $A^{e}$ and $B^{e}((15)$ and (16)) can be written as

$$
\begin{aligned}
& A^{e}(r, \theta, \phi)= A_{0}(r, \theta, \phi)+(1-\lambda) A_{S F}^{*}(r, \theta, \phi)+\lambda A_{R}^{*}(r, \theta, \phi) \\
& B^{e}(r, \theta, \phi)=B_{0}(r, \theta, \phi)+(1-\lambda) B_{S F}^{*}(r, \theta, \phi)+\lambda B_{R}^{*}(r, \theta, \phi) \\
&+3(1-\lambda) \frac{r}{a^{3}}\left[\int_{0}^{a^{2} / r} R B_{0}(R, \theta, \phi) d R\right. \\
& \\
&\left.\quad-(1-2 \lambda) \frac{a^{6 \lambda}}{r^{3 \lambda}} \int_{0}^{a^{2} / r} R^{1-3 \lambda} B_{0}(R, \theta, \phi) d R\right],
\end{aligned}
$$

where

$$
A_{S F}^{*}(r, \theta, \phi)=-\frac{r}{a} A_{0}\left(\frac{a^{2}}{r}, \theta, \phi\right),
$$




$$
\begin{aligned}
A_{R}^{*}(r, \theta, \phi)= & -\frac{\left(r^{2}+a^{2}\right)}{2 a r} A_{0}\left(\frac{a^{2}}{r}, \theta, \phi\right)+\frac{\left(r^{2}-a^{2}\right)}{a} \frac{\partial}{\partial r} A_{0}\left(\frac{a^{2}}{r}, \theta, \phi\right) \\
& -\frac{r^{2}\left(r^{2}-a^{2}\right)^{2}}{4 a^{3}} \nabla^{2}\left(r^{-1} A_{0}\left(\frac{a^{2}}{r}, \theta, \phi\right)\right), \\
B_{S F}^{*}(r, \theta, \phi)= & \frac{a}{r} B_{0}\left(\frac{a^{2}}{r}, \theta ; \phi\right)-\frac{3 r}{a^{3}} \int_{0}^{a^{2} / r} R B_{0}(R, \theta, \phi) d R, \\
B_{R}^{*}(r, \theta, \phi)= & -\frac{a}{r} B_{0}\left(\frac{a^{2}}{r}, \theta, \phi\right),
\end{aligned}
$$

are the perturbed terms due to shear-free (suffix $S F$ ) and rigid (suffix $R$ ) spheres $[13,12]$ respectively.

COROLlaRY. Assuming $A^{e}(r, \theta, \phi)=A^{e}(r, \theta), A^{i}(r, \theta, \phi)=A^{i}(r, \theta)$, $B^{e}(r, \theta, \phi)=B^{i}(r, \theta, \phi)=0$ in (15)-(18) and differentiating with respect to $\theta$, and setting $\partial A^{e} / \partial \theta=\psi^{e} / r \sin \theta, \partial A^{i} / \partial \theta=\psi^{i} / r \sin \theta, \partial A_{0} / \partial \theta=$ $\psi_{0} / r \sin \theta$ we obtain the theorem for axisymmetric Stokes flow past a fluid sphere in terms of the Stokes stream function $\psi(r, \theta)$. The corresponding solution for the motion exterior to the fluid sphere is given by

$$
\begin{aligned}
& \psi^{e}(r, \theta)= \psi_{0}(r, \theta)+(1-\lambda)\left[-\frac{r^{3}}{a^{3}} \psi_{0}\left(\frac{a^{2}}{r}, \theta\right)\right] \\
&+\lambda\left[\frac{r\left(r^{2}-3 a^{2}\right)}{2 a^{3}} \psi_{0}\left(\frac{a^{2}}{r}, \theta\right)+\frac{r^{2}\left(r^{2}-a^{2}\right)}{a^{3}} \frac{\partial}{\partial r} \psi_{0}\left(\frac{a^{2}}{r}, \theta\right)\right. \\
&\left.-\frac{r^{2}\left(r^{2}-a^{2}\right)^{2}}{4 a^{5}} D^{2}\left(r \psi_{0}\left(\frac{a^{2}}{r}, \theta\right)\right)\right]
\end{aligned}
$$

and for the motion interior to the fluid sphere

$$
\begin{gathered}
\psi^{i}(r, \theta)=(1-\lambda)\left[\frac{3\left(a^{2}-r^{2}\right)}{2 a^{2}} \psi_{0}(r, \theta)+\frac{r\left(r^{2}-a^{2}\right)}{a^{2}} \frac{\partial}{\partial r} \psi_{0}(r, \theta)\right. \\
\left.-\frac{\left(r^{2}-a^{2}\right)^{2}}{4 a^{2}} D^{2} \psi_{0}(r, \theta)\right]
\end{gathered}
$$

where $\psi(r, \theta)$ satisfies $D^{4} \psi=0$ with

$$
D^{2}=\frac{\partial^{2}}{\partial r^{2}}+\frac{\sin \theta}{r^{2}} \frac{\partial}{\partial \theta} \frac{1}{\sin \theta} \frac{\partial}{\partial \theta}
$$




\section{Deductions}

Rigid sphere Setting $\lambda=1$ in (15) and (16) and noting that $A^{i}=B^{i}=O$ we obtain the theorem for non-axisymmetric Stokes flow past a rigid sphere [13]. Similarly, by setting $\lambda=1$ in (21) we recover Collins' [2] sphere theorem for axisymmetric flow.

Shear-free sphere Setting $\lambda=a$ in (15) and (16) we get the theorem for non-axisymmetric Stokes flow past a shear-free sphere [12], and setting $\lambda=0$ in (21) we obtain Harper's theorem [7].

\section{Drag on the fluid sphere}

The expression for the drag on the fluid sphere is [8]

$$
\mathbf{F}=2 \pi \mu^{e} a(2+\lambda)\left[\mathbf{V}_{0}\right]_{0}+\pi \mu^{e} a^{3} \lambda\left[\nabla^{2} \mathbf{V}_{0}\right]_{\mathbf{o}}
$$

where $\mathbf{V}_{0}$ is the velocity due to the undisturbed flow and the subscript $o$ indicates evaluation of the quantities inside the square brackets at the centre of the sphere. The equation (23) can be written as

$$
\mathbf{F}=(1-\lambda) \mathbf{F}_{S F}+\lambda F_{R},
$$

where

$$
\mathbf{F}_{S F}=4 \pi \mu^{e} a\left[\mathbf{V}_{0}\right]_{\mathbf{o}}, \quad \mathbf{F}_{R}=6 \pi \mu^{e} a\left[\mathbf{V}_{0}\right]_{\mathbf{o}}+\pi \mu^{e} a^{3}\left[\nabla^{2} \mathbf{V}_{0}\right]_{\mathbf{o}}
$$

are the forces on the shear-free and rigid spheres respectively. When $\lambda=1$, we get the force acting on a rigid spherical particle placed in an arbitrary Stokes flow (Faxen's law) and when $\lambda=0$, we recover the force acting on a shear-free sphere. Note that the torque on the fluid sphere is zero. This is due to the continuity of tangential stresses across the surface of the liquid drop.

\section{Examples}

\subsection{Stokeslet outside the liquid sphere, perpendicular to the radius vector:} Consider a Stokeslet exerting a force $\left(F_{1}, 0,0\right)$ at the point $(0,0, c), c>a$. The corresponding expressions for $A_{0}$ and $B_{0}$ due to this Stokeslet in an unbounded fluid are [13] 


$$
\begin{aligned}
& A_{0}(r, \theta, \phi)=\frac{F_{1}}{8 \Pi \mu}\left(\frac{(r \cos \theta-c)}{c} R_{1}+\frac{R_{1}^{2}}{c}\right) \frac{\cos \phi}{r \sin \theta}, \\
& B_{0}(r, \theta, \phi)=\frac{F_{1}}{4 \Pi \mu}\left(R_{1}+r \cos \theta-c\right) \frac{\sin \phi}{r c \sin \theta},
\end{aligned}
$$

where $R_{1}^{2}=r^{2}-2 r c \cos \theta+c^{2}$.

Applying the theorem we obtain the exterior and interior flow fields as

$$
\begin{aligned}
A^{e}(r, \theta, \phi)=\frac{F_{1}}{8 \Pi \mu} & \left(\frac{(r \cos \theta-c)}{c} R_{1}+\frac{R_{1}^{2}}{c}\right) \frac{\cos \phi}{r \sin \theta} \\
& +\lambda\left\{-\frac{\left(r^{2}+a^{2}\right) c \cos \phi}{2 a^{3} r^{2} \sin \theta}\left(R_{2}^{2}-\left(r-\frac{a^{2}}{c} \cos \theta\right) R_{2}\right)\right\} \\
+ & \lambda\left\{\frac{\left(r^{2}-a^{2}\right) \cos \phi}{a^{3} r \sin \theta}\right. \\
& \left.\times\left(\frac{c}{r}\left(r^{2}-\frac{a^{4}}{c^{2}}\right)-\frac{a^{2}}{r} R_{2} \cos \theta-\frac{c\left(r-\frac{a^{2}}{c} \cos \theta\right)^{2}}{R_{2}}\right)\right\} \\
& +\lambda\left\{\frac{\left(r^{2}-a^{2}\right)^{2} \cos \phi}{2 a c R_{2} r^{2} \sin \theta}\left(r-\frac{a^{2}}{c} \cos \theta-R_{2}\right)\right\} \\
& \left.+(1-\lambda)\left\{\frac{c \cos \phi}{a^{3} \sin \theta}\left(\left(r-\frac{a^{2}}{c} \cos \theta\right) R_{2}-R_{2}^{2}\right)\right\}\right]
\end{aligned}
$$

$B^{e}(r, \theta, \phi)=\frac{F_{1}}{4 \Pi \mu}\left\{\left(R_{1}+r \cos \theta-c\right) \frac{\sin \phi}{r c \sin \theta}\right.$

$$
\begin{aligned}
& +\lambda\left\{-\frac{\sin \phi}{a r \sin \theta}\left(R_{2}+\frac{a^{2}}{c} \cos \theta-r\right)\right\} \\
& +(1-\lambda)\left\{\frac{\sin \phi}{a r \sin \theta}\left(R_{2}+\frac{a^{2}}{c} \cos \theta-r\right)\right. \\
& \left.-\frac{3 r \sin \phi}{a^{3} c \sin \theta}\left(\frac{I}{2}+\frac{a^{2}}{r}\left[\frac{a^{2}}{2 r} \cos \theta-c\right]\right)\right\} \\
& +3(1-\lambda) \frac{r \sin \phi}{a^{3} c \sin \theta}\left\{\left(\frac{I}{2}+\frac{a^{2}}{r}\left[\frac{a^{2}}{2 r} \cos \theta-c\right]\right)\right. \\
& -(1-2 \lambda) \frac{a^{6 \lambda}}{r^{3 \lambda}}\left(\int_{0}^{a^{2} / r} R^{-3 \lambda}\left(R^{2}-2 c R \cos \theta+c^{2}\right)^{1 / 2} d R\right. \\
& \left.\left.\left.+\frac{a^{2-6 \lambda}}{r^{1-3 \lambda}}\left[\frac{a^{2} \cos \theta}{r(2-3 \lambda)}-\frac{c}{(1-3 \lambda)}\right]\right)\right\}\right\}
\end{aligned}
$$


where

$$
\begin{aligned}
I= & \left(\frac{a^{2}}{c}-r \cos \theta\right) \frac{c^{2}}{r^{2}} R_{2}+c^{2} \cos \theta \\
& +c^{2} \sin ^{2} \theta\left[\log \left(\frac{a^{2}}{r}-c \cos \theta+\frac{c}{r} R_{2}\right)-\log c(1-\cos \theta)\right], \\
R_{2}^{2}= & r^{2}-2 \frac{a^{2}}{c} r \cos \theta+\frac{a^{4}}{c^{2}} \\
A^{i}(r, \theta, \phi)= & \frac{F_{1}}{8 \Pi \mu}(1-\lambda) \frac{1}{\sin \theta}\left[\frac{\left(a^{2}-r^{2}\right)}{2 a^{2} r}\left(\frac{(r \cos \theta-c)}{c} R_{1}+\frac{R_{1}^{2}}{c}\right) \cos \phi\right. \\
& +\frac{r\left(r^{2}-a^{2}\right)}{a^{2}} \frac{\partial}{\partial r}\left(\frac{(r \cos \theta-c)}{r c} R_{1}+\frac{R_{1}^{2}}{r c}\right) \cos \phi \\
& \left.\quad-\frac{\left(r^{2}-a^{2}\right)^{2}}{4 a^{2}} \nabla^{2}\left(\frac{(r \cos \theta-c)}{r c} R_{1}+\frac{R_{1}^{2}}{r c}\right) \cos \phi\right] \\
B^{i}(r, \theta, \phi)= & \frac{F_{1}}{4 \Pi \mu}(1-\lambda) \frac{\sin \phi}{c \sin \theta}\left[\frac{2}{r}\left(R_{1}+r \cos \theta-c\right)\right. \\
& \quad 3(1-2 \lambda) r^{3 \lambda-2}\left(\int_{0}^{r} R^{-3 \lambda}\left(R^{2}-2 c R \cos \theta+c^{2}\right)^{1 / 2} d R\right. \\
& \left.\left.+r^{1-3 \lambda}\left[\frac{r \cos \theta}{2-3 \lambda}-\frac{c}{1-3 \lambda}\right]\right)\right]
\end{aligned}
$$

This problem was also discussed by Fuentes [4] by making use of Lamb's solutions. However the above analysis is simpler and the solution is in a closed form. For this reason, the method proposed in Section 3 is more efficient. The corresponding axisymmetric case was also discussed by Fuentes [3]. It may be noted that these results can be easily obtained by using (21) and (22). The drag on the fluid sphere may be calculated as explained in [13] and is given by

$$
\mathbf{F}=\frac{F_{1}}{4}\left(\lambda\left(\frac{3 a}{c}+\frac{a^{3}}{c^{3}}\right)+(1-\lambda) \frac{2 a}{c}\right) \hat{\mathbf{i}} .
$$

When $\lambda=1$ in (33) we recover the expression for drag in the case of a rigid sphere. This agrees with the result of Higdon [9] who has used Oseen's [11] image solution to model the motion of micro-organisms by flagellar propulsion.

6.2. Rotlet at $(0,0, c), c>a$ : Consider a rotlet of strength $\left(0, F_{2}, 0\right)$ located at $(0,0, c)$. The corresponding expressions for $A_{0}$ and $B_{0}$ in an unbounded fluid 
are

$$
\begin{aligned}
& A_{0}(r, \theta, \phi)=\frac{F_{2}}{8 \Pi \mu}\left[-R_{1}+c-r \cos \theta\right] \frac{\cos \phi}{r c \sin \theta} \\
& B_{0}(r, \theta, \phi)=\frac{F_{2}}{8 \Pi \mu}\left(\frac{c-r \cos \theta}{R_{1}}-1\right) \frac{\sin \phi}{r c \sin \theta}
\end{aligned}
$$

From the theorem

$$
\begin{aligned}
& A^{e}(r, \theta, \phi)=\frac{F_{2}}{8 \Pi \mu}\left\{\left[-R_{1}+c-r \cos \theta\right] \frac{\cos \phi}{r c \sin \theta}\right. \\
& +(1-\lambda)\left[c R_{2}-r c+a^{2} \cos \theta\right] \frac{r \cos \phi}{c a^{3} \sin \theta} \\
& +\lambda\left(\frac{\left(r^{2}+a^{2}\right)}{2 r}\left[c R_{2}-r c+a^{2} \cos \theta\right]\right. \\
& \left.\left.-\left(r^{2}-a^{2}\right) \frac{\partial}{\partial r}\left[c R_{2}-r c+a^{2} \cos \theta\right]\right) \frac{\cos \phi}{c a^{3} \sin \theta}\right\}, \\
& B^{e}(r, \theta, \phi)=\frac{F_{2}}{8 \Pi \mu}\left[\left(\frac{c-r \cos \theta}{R_{1}}-1\right) \frac{\sin \phi}{r c \sin \theta}\right. \\
& -\lambda\left(\frac{\left(r-\frac{a^{2}}{c} \cos \theta\right)}{R_{2}}-1\right) \frac{\sin \phi}{a c \sin \theta} \\
& +(1-\lambda)\left(\frac{\left(r-\frac{a^{2}}{c} \cos \theta\right)}{R_{2}}+2-\frac{3 r}{2 a^{2}} \frac{\partial I}{\partial c}\right) \frac{\sin \phi}{a c \sin \theta} \\
& +3(1-\lambda)\left(\frac{r}{2 a^{2} c} \frac{\partial I}{\partial c}-\frac{1}{c}\right) \frac{\sin \phi}{a \sin \theta} \\
& -3(1-\lambda)(1-2 \lambda) \frac{a^{6 \lambda-3}}{r^{3 \lambda-1}} \\
& \left.\times\left\{\int_{0}^{a^{2} / r}\left[\frac{(c-R \cos \theta)}{\left(R^{2}-2 c R \cos \theta+c^{2}\right)^{1 / 2}}-1\right] R^{-3 \lambda} d R\right\} \frac{\sin \phi}{c \sin \theta}\right] \\
& A^{i}(r, \theta, \phi)=\frac{F_{2}}{8 \Pi \mu}(1-\lambda)\left\{\frac{\left(a^{2}-r^{2}\right)}{2 a^{2} r}\left[-R_{1}+c-r \cos \theta\right]\right. \\
& \left.+\frac{r\left(r^{2}-a^{2}\right)}{a^{2}} \frac{\partial}{\partial r}\left(\frac{-R_{1}+c-r \cos \theta}{r}\right)\right\} \frac{\cos \phi}{c \sin \theta}
\end{aligned}
$$




$$
\begin{aligned}
& B^{i}(r, \theta, \phi)=\frac{F_{2}}{8 \Pi \mu}(1-\lambda) \frac{\sin \phi}{c \sin \theta}\left\{2\left(\frac{(c-r \cos \theta)}{r R_{1}}-\frac{1}{r}\right)\right. \\
&-3(1-2 \lambda) r^{3 \lambda-2} \\
&\left.\times \int_{0}^{r} R^{-3 \lambda}\left[\frac{(c-r \cos \theta)}{\left(R^{2}-2 c R \cos \theta+c^{2}\right)^{1 / 2}}-1\right] d R\right\} .
\end{aligned}
$$

The drag on the fluid sphere is found to be

$$
\mathbf{F}=\left(\frac{3}{4} \lambda+\frac{(1-\lambda)}{2}\right) F_{2} \frac{a}{c^{2}} \hat{\mathbf{i}} .
$$

To the best of the authors knowledge, this result was not available in literature.

6.3. Potential doublet at $(0,0, c), c>a$ : Consider a potential-doublet of strength $\left(S_{1}, 0,0\right)$ located at $(0,0, c)$. The corresponding expressions for $A_{0}$ and $B_{0}$ are

$$
\begin{aligned}
A_{0} & =\frac{S_{1}}{6 \Pi \mu}\left(\frac{c-r \cos \theta}{R_{1}}-1\right) \frac{\cos \phi}{r c \sin \theta}, \\
B_{0} & =0 .
\end{aligned}
$$

Applying the theorem we obtain

$$
\begin{aligned}
A^{e}=\frac{S_{1}}{6 \Pi \mu} & \frac{\cos \phi}{r c \sin \theta} \\
\times\left\{\left(\frac{c-r \cos \theta}{R_{1}}-1\right)-(1-\lambda) \frac{r^{3}}{a^{3}}\left(\frac{\left(r-\frac{a^{2}}{c} \cos \theta\right)}{R_{2}}-1\right)\right. & -\lambda \frac{r\left(r^{2}+a^{2}\right)}{2 a^{3}}\left(\frac{\left(r-\frac{a^{2}}{c} \cos \theta\right)}{R_{2}}-1\right) \\
& \left.+\lambda \frac{r\left(r^{2}-a^{2}\right)}{a^{3}} \frac{\partial}{\partial r}\left(\frac{r\left(r-\frac{a^{2}}{c} \cos \theta\right)}{R_{2}}-r\right)\right\}, \\
A^{i}=\frac{S_{1}}{6 \Pi \mu}(1-\lambda) \frac{\cos \phi}{r c \sin \theta}\left\{\frac{\left(a^{2}-r^{2}\right)}{2 a^{2}}\left(\frac{c-r \cos \theta}{R_{1}}-1\right)\right. & \left.+\frac{r^{2}\left(r^{2}-a^{2}\right)}{a^{2}} \frac{\partial}{\partial r}\left(\frac{c-r \cos \theta}{r R_{1}}-\frac{1}{r}\right)\right\} .
\end{aligned}
$$

The drag in this case is

$$
\mathbf{F}=-(3 \lambda+2(1-\lambda)) S_{1} \frac{a}{3 c^{3}} \hat{\mathbf{i}} .
$$

The authors believe that this is a new result. 
6.4. Stokes-doublet at $(0,0, c)$ : The expressions for $A_{0}$ and $B_{0}$ for a Stokesdoublet of strength $M$ in an unbounded fluid are (refer to (31) and (32) of [13])

$$
\begin{gathered}
A_{0}(r, \theta, \phi)=\frac{M}{16 \Pi \mu}\left[\frac{2(r-c \cos \theta)}{r c^{2} \sin ^{2} \theta} R_{1}-\frac{r(r-c \cos \theta)}{c^{2} R_{1}}\right. \\
\left.\quad+\frac{2}{r c \sin ^{2} \theta}\left(r-c \cos \theta+r \cos ^{2} \theta\right)\right] \sin 2 \phi, \\
B_{0}(r, \theta, \phi)=\frac{M}{8 \Pi \mu}\left\{\left[\frac{-2 \cos \theta}{r c^{2} \sin ^{2} \theta} R_{1}+\frac{1}{c R_{1}}-\frac{2 \cos \theta}{r c \sin ^{2} \theta}\right] \cos 2 \phi-\frac{1}{c R_{1}}\right\} .
\end{gathered}
$$

The exterior and interior flow fields can be obtained by applying the theorem (Section 3). It is found that the drag on the fluid sphere in this case is zero.

\section{Acknowledgement}

The authors are grateful to the referees whose constructive suggestions have led to the present improved version.

\section{References}

[1] S. F. J. Butler, "A note on Stokes stream function for the motion of a spherical boundary", Proc. Camb. Phil. Soc. 49 (1953) 168-174.

[2] W. D. Collins, "Note on a sphere theorem for the axisymmetric Stokes flow of a viscous fluid", Mathematika 5 (1958) 118-121.

[3] Y. O. Fuentes, S. Kim and D. J. Jeffrey, "Mobility functions for two unequal viscous drops in Stokes flow I Axisymmetric motions", Phy. Fluids 31 (1988) 2445-2455.

[4] Y. O. Fuentes, S. Kim and D. J. Jeffrey, "Mobility functions for two unequal viscous drops in Stokes flow II Axisymmetric motions", Phy. Fluids A 1 (1989) 61-76.

[5] W. Hackborn, M. E. O'Neill and K. B. Ranger, "The structure of an asymmetric Stokes flow", Q.J. Mech. Appl. Math. 39 (1986) 1-14.

[6] J. S. Hadamard, Compt. Rend. Acad. Sci. (Paris) 152 and 154 (1911 and 1912) 1735 and 109.

[7] J. F. Harper, "Axisymmetric Stokes flow images in spherical free surfaces with applications to rising bubbles", J. Aust. Math. Soc. Ser. B 25 (1983) 217-231.

[8] G. Hetsroni and S. Haber, "The flow in and around a droplet or bubble submerged in an unbound arbitrary velocity field", Rheol. Acta 9 (1970) 488-496.

[9] J. J. L. Higdon, "A hydrodynamic analysis of flagellar propulsion", J. Fluid Mech. 90 (1979) 685-711.

[10] H. Lamb, Hydrodynamics, (Dover, 1945) 596. 
[11] C. W. Oseen, Hydrodynamik (Acad. Verlag, Leipzig, 1927).

[12] D. Palaniappan, S. D. Nigam, T. Amaranath and R. Usha, "A theorem for a shear-free sphere in Stokes flow", Mech. Res. Comm. 17 (1990) 429-435.

[13] D. Palaniappan, S. D. Nigam, T. Amaranath and R. Usha, "Lamb's solution of the Stokes equations - A sphere theorem", Q. J. Mech. Appl. Math. 45 (1992) 47-56.

[14] J. M. Rallison, "Note on Faxen relations for a particle in Stokes flow", J. Fluid Mech. 88 (1978) 529-533.

[15] W. Rybczynski, Bull. Acad. Sci. Cracovie Ser. A (1911) 40.

[16] R. Shail, "A note on some asymmetric Stokes flows within a sphere", Q. J. Mech. Appl. Math. 40 (1987) 223-233.

[17] R. Shail and S. H. Onslow, "Some Stokes flows exterior to a spherical boundary", Mathematika 35 (1988) 233-246. 